J. Clin. Chem. Clin. Biochem.

Vol. 27, 1989, pp. 27-31

(C) 1989 Walter de Gruyter \& Co. Berlin - New York

\title{
Fractionation of Urinary 17-Ketosteroids by Gas Chromatography: A Neglected Procedure in the Assessment of Hyperandrogenicity in the Hirsute Female
}

\author{
By M. S. Shapiro, S. Zelefsky
}

Endocrine Unit Department of Medicine C Meir Hospital Kfar Sava

R. Chayen

Endocrine Laboratory Ichilov Hospital Tel-Aviv and

M. M. Werber

Department of Medical Laboratories Meir Hospital Kfar Sava

(Received April 6/October 14, 1988)

Summary: $17-$ Ketosteroids were determined by gas chromatography in twenty four-hour urine samples from 62 hirsute females. The method permitted the determination of androsterone (A), aetiocholanolone (E) and dehydroepiandrosterone (D). Elevated concentrations of one or more of these metabolites were detected in $81 \%$ of the samples.

Two main patterns of hyperandrogenicity were observed:

1) Hyper $A+E(27 \%)$ and

2) Hyper A (26\%);

Elevated $\mathrm{AD}, \mathrm{AED}, \mathrm{D}, \mathrm{ED}$ or $\mathrm{E}$ were less common, but in total these patterns comprised another $28 \%$.

The plasma testosterone and total urinary 17 -ketosteroid concentrations were elevated in only $21 \%$ and $23 \%$ of the samples, respectively.

Thirty two out of 33 patients with elevated urine metabolites showed significant suppression following dexamethasone administration ( $2 \mathrm{mg}$ /day during 6 days). Thus, dexamethasone suppressable hyperandrogenicity was predominant in this group of hirsute females. Elevations of urinary androsterone and aetiocholanolone are probably contingent on the relative activities of $5 \alpha$ - and $5 \beta$-reductases $\left.{ }^{1}\right)$ in the presence of increased androstenedione secretion. Elevations of urinary dehydroepiandrosterone suggest decreased adrenal cortical $3 \beta$-hydroxysteroid dehydrogenase ${ }^{1}$ ) activity. Thus, fractionation of urinary 17 -ketosteroids seems to be an effective test in the evaluation of hirsutism.

1) Enzymes

$3 \alpha$-Hydroxysteroid dehydrogenase:

$3 \alpha$-Hydroxysteroid: NAD(P) ${ }^{+}$-oxidoreductase (EC 1.1.1.50)

$3 \beta$-Hydroxysteroid dehydrogenase:

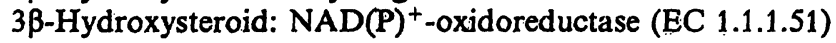

$5 \alpha$-Reductase:

$3 \alpha$-Hydroxy-5 $\alpha$-steroid:NAD ${ }^{+}-3$-oxidoreductase

(EC 1.1.1.151)

5ß-Reductase:

$3 \alpha$-Hydroxy-5 $\beta$-steroid:NAD+-3-oxidoreductase

(EC 1.1.1.152)

\section{Introduction}

A relatively undervalued diagnostic tool for establishing the presence of excess production of androgens is fractionation of the urinary 17-ketosteroids, with measurement of androsterone, aetiocholanolone, and dehydroepiandrosterone. The usefulness of this technique in evaluating patients with clomiphene-resistant anovulation has been previously reported (1). 
We have now used this procedure in conjunction with dexamethasone and human chorionic gonadotropin administration in the evaluation of females with predominant hirsutism.

Several different patterns of hyperandrogenicity were detected involving different combinations of the three androgens androsterone, aetiocholanolone or dehydroepiandrosterone. This report presents and discusses the significance of these fractionation patterns as applied to the urinary $17-$ ketosteroids of 62 patients with hirsutism, 33 of whom also completed the combined hormone administration regimen.

\section{Materials and Methods}

\section{Patients population}

Sixty two hirsute females (age range 20-35 years) were examined in the endocrine outpatient clinic. Thirty had associated menstrual disorders, and 18 had acne. Only one patient complained of difficulties with conception. Hirsutism was graded in 4 stages (2).

\section{Laboratory methods}

The 24-hour urinary 17-ketosteroids were measured by a chemical technique (3). For determination of androsterone, aetiocholanolone and dehydroepiandrosterone, urinary 17-ketosteroids were fractionated by gas chromatography by the method of Gould et al. (4). Plasma concentrations of hormones were measured using commercial radioimmunoassay kits: testosterone - Serono Diagnostics; prolactin, luteinizing hormone and follicle stimulating hormone - International CIA Sorin (Saluggia, Cedex).

All data are given as mean \pm standard error mean.

\section{Dynamic endocrine tests}

Dexamethasone was administered orally $(2 \mathrm{mg} /$ day during 6 days). Human chorionic gonadotropin, 2500 units, was injected on days 4,5 and 6 . The urinary $17-$ ketosteroids were fractionated before dexamethasone administration, on day 3 of dexamethasone administration and on day 6 during dexamethasone and human chorionic gonadotropin administration (5). This procedure was completed for thirty six patients.

$$
: 1
$$

\section{Results}

Fifty of the 62 females (81\%) showed elevations of one or more of the individual androgens (androsterone), (aetiocholanolone) or (dehydroepiandrosterone) (references values: androsterone $2.1 \pm 0.7 \mathrm{mg} / 24 \mathrm{~h}$; aetiocholanolone $1.9 \pm 0.8 \mathrm{mg} / 24 \mathrm{~h}$; dehydroepiandrosterone $0.9 \pm 0.3 \mathrm{mg} / 24 \mathrm{~h}$ ).

Two main patterns of abnormal androgen secretion were observed:

1) Hyper $\mathrm{AE}, 1 \overline{7}$ patients (27\%): androsterone 5.4 $\pm 0.4 \mathrm{mg} / 24 \mathrm{~h}$; aetiocholanolone $3.8 \pm 0.4 \mathrm{mg} / 24 \mathrm{~h}$;

2) Hyper A, 16 patients (26\%): androsterone 4.4 $\pm 0.3 \mathrm{mg} / 24 \mathrm{~h}$ (fig. 1 ).

Other minor patterns observed were:

$\mathrm{AD}, 5$ patients $(8 \%)$ : androsterone $5.6 \pm 1.0 \mathrm{mg} /$ $24 \mathrm{~h}$;

dehydroepiandrosterone $2.1 \pm 0.4 \mathrm{mg} / 24 \mathrm{~h}$;

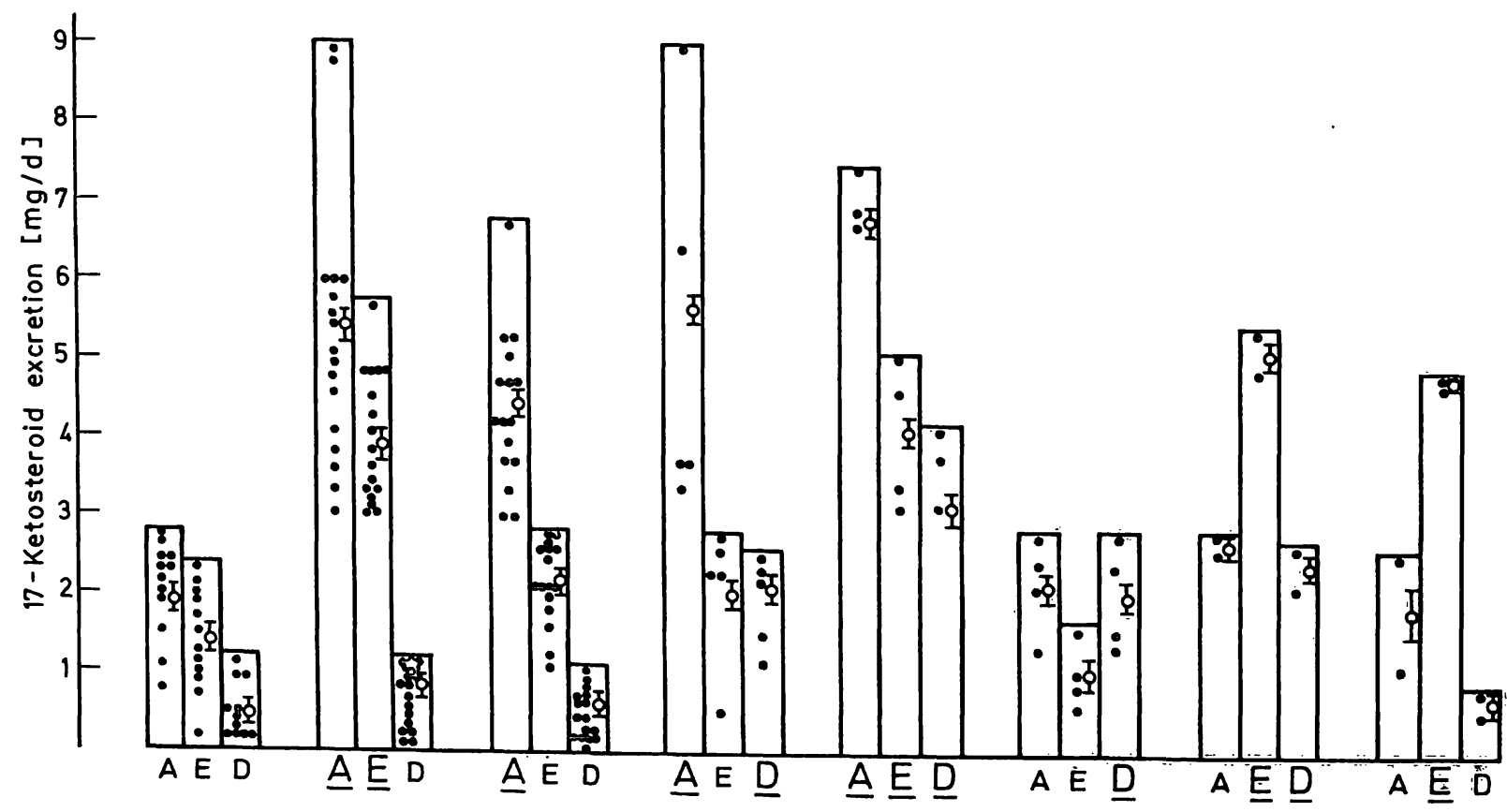

Fig. 1. Fractionation of urinary 17-ketosteroids; androsterone (A), aetiocholanolone (E) and dehydroepiandrosterone (D). The first panel represents the upper limits of normal in non-hirsute patients. The individual points in this panel represent the values (in $\mathrm{mg} / 24 \mathrm{~h}$ ) in 12 out of 62 hirsute patients with normal concentrations of $\mathrm{A}, \mathrm{E}$ and $\mathrm{D}$. The remaining seven panels represent 7 groups of hyperandrogenicity noted in 50 out of 62 patients with their respective individual levels of $A, E$, and $D$. The groups are listed in descending order of frequency. The mean \pm standard error mean is included for each androgen in every panel. 
AED: androsterone $6.7 \pm 0.2 \mathrm{mg} / 24 \mathrm{~h}$; aetiocholanolone $4.1 \pm 0.5 \mathrm{mg} / 24 \mathrm{~h}$; dehydroepiandrosterone 3.1 $\pm 0.5 \mathrm{mg} / 24 \mathrm{~h}$ and

$\mathrm{D}$ : dehydroepiandrosterone $2.6 \pm 0.4 \mathrm{mg} / 24 \mathrm{~h}$ with four patients $(6 \%)$ each;

ED: aetiocholanolone $5.1 \pm 0.1 \mathrm{mg} / 24 \mathrm{~h}$; dehydroepiandrosterone $2.35 \pm 0.1 \mathrm{mg} / 24 \mathrm{~h}$ and

E: aetiocholanolone $4.75 \pm 0.1 \mathrm{mg} / 24 \mathrm{~h}$, with two patients ( $3 \%$ ) each (fig. 1).

The extent of the increase of androgen concentration did not correlate with the stage of hirsutism, as previously reported by other workers (5).

In contrast, the total urinary $17-$ ketosteroids were elevated in only 14 patients (23\%). Plasma testosterone was mildly elevated in 13 patients $(21 \%)$ with concentrations of $13 \pm 4 \mathrm{ng} / 1$ (normal: $<10 \mathrm{ng} / \mathrm{l}$ ). Plasma prolactin, luteinizing hormone and follicle stimulating hormone concentrations were appropriate for the respective menstrual states in all patients.

Thirty two out of $33(97 \%)$ patients receiving both dexamethasone and human chorionic gonadotropin showed significant suppression of the elevated androgen concentrations following dexamethasone admin- istration. In the Hyper AE group (12 patients), the concentration of androsterone dropped to $1.5 \pm 0.19$ $\mathrm{mg} / 24 \mathrm{~h}$ and that of aetiocholanolone to $0.92 \pm 0.1$ $\mathrm{mg} / 24 \mathrm{~h}$ after dexamethasone (fig. 2). The androsterone concentrations of the Hyper A group (10 patients) fell to $1.2 \pm 0.16 \mathrm{mg} / 24 \mathrm{~h}$. The 10 remaining patients in the other groups who were receiving dexamethasone all showed significant suppression of their respective elevated androgens (fig. 2).

Two patients in the Hyper AE group showed elevations of $50 \%$ or more of both androsterone and aetiocholanolone following administration of human chorionic gonadotropin. In a single patient with $\mathrm{Hy}-$ per A, the level of androsterone was not suppressed with dexamethasone, and it rose from 3.9 to $6.1 \mathrm{mg} /$ $24 \mathrm{~h}$ following chorionic gonadotropin administration.

\section{Discussion}

Fractionation of 17-ketosteroids demonstrated the presence of hyperandrogenicity in $81 \%$ of the patients, whereas total urinary $17-$ ketosteroids and plasma testosterone levels were elevated in only $23 \%$ and $21 \%$, respectively.
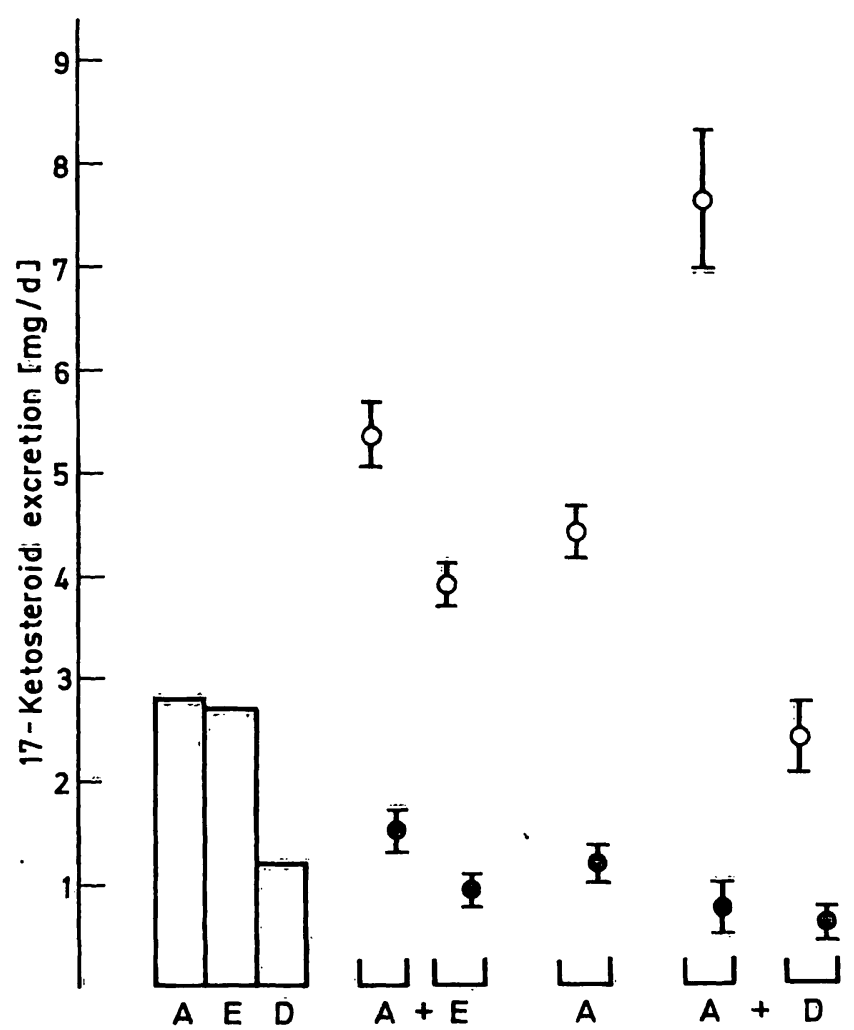

$\Phi$

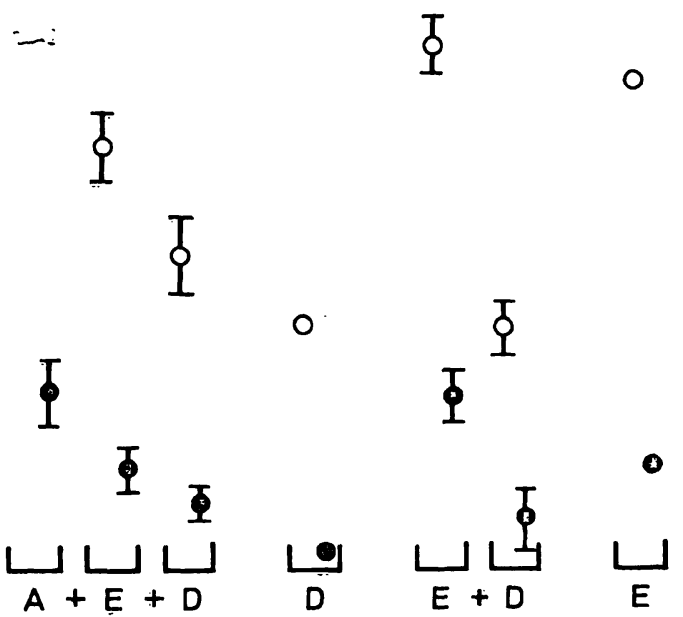

Fig. 2. Fractionation of urinary 17-ketosteroids: androsterone (A), aetiocholanolone (E) and dehydroepiandrosterone (D) with dexamethasone suppression. The first panel represents the upper limits of normal in non-hirsute patients (as in fig. 1). Androgen levels before $(0)$ and following $(\bullet)$ administration of dexamethasone $2 \mathrm{mg} /$ day for 6 days are presented for each group (21 patients). The mean \pm standard error mean are listed for Groups AE (12 patients); A (10 patients); AD (2 patients); AED (4 patients) and ED (2 patients). In Groups $\mathrm{D}$ and $\mathrm{E}$ individual points represent single patients. 
The varying patterns of hyperandrogenicity detected by gas chromatography in hirsute females are of interest. Dehydropiandrosterone is predominantly of adrenal cortical origin (6). The levels of urinary dehydroepiandrosterone are inversely proportional to adrenal cortical $3 \beta$-hydroxysteroid dehydrogenase activity. Thus, elevation of dehydroepiandrosterone suggests reduced adrenal $3 \beta$-hydroxysteroid dehydrogenase activity $(7,8)$. Bongiovanni $(8)$ has also demonstrated that mild deficiencies of $3 \beta$-hydroxysteroid dehydrogenase may be common in hirsute women. Fifteen of our patients $(24 \%)$ showed elevated dehydroepiandrosterone, suggesting an adrenal origin for this metabolite (fig. 1).

The origin of the elevated urinary androsterone and aetiocholanolone remains controversial. Both androsterone and aetiocholanolone are derived predominantly from androstenedione (9). Androstenedione is secreted from both the adrenal cortex and the ovary $(6,10)$.

Testosterone and androstenedione are metabolized by reduction of the $\mathrm{C}_{3}$ ketone group (mainly by $3 \alpha-$ hydroxysteroid dehydrogenase) and the $\mathrm{C}_{4}$-ene by $5 \alpha$ and $5 \beta$-reductases (9).

The $3 \alpha-5 \alpha$-derivative is androsterone, whereas the $3 \alpha$ $5 \beta$-derivative is aetiocholanolone. Variations in the relative activity of the $5 \alpha$ - and $5 \beta$-reductases will be reflected in the relative amounts of androsterone and aetiocholanolone secreted.

The Hyper A pattern suggests enhanced $5 \alpha$-reductase activity, while the Hyper E pattern suggests enhanced $5 \beta$-reductase activity; in the Hyper AE pattern the activities of the $5 \alpha$ - and $5 \beta$-reductases are almost comparable.

The Hyper A pattern was seen in $16(26 \%)$ and the Hyper E pattern in $2(3 \%)$ of the patients. Elevations of androsterone, alone or in combination with other metabolites, were seen in 42 patients $(68 \%)$. Similarly, aetiocholanolone was elevated in a total of 25 patients $(40 \%)$. An increase in $5 \alpha$-reductase activity appears, therefore, to be a factor to be taken into account in a significant proportion of these hirsute women. Indeed, elevated $5 \alpha$-reductase activity has been demonstrated in the skin of hirsute females (11).

Many studies have presented evidence for either an adrenal $(8,12,13)$, ovarian $(14-17)$ or mixed ovarian-adrenal (18) source of androgen production in hirsute patients. The most convincing evidence for ovarian suppression with dexamethasone administration was derived from ovarian and adrenal venous catheterization studies (16). Although concentrations of testosterone and androstenedione were higher in the ovarian veins, 20 out of 44 patients exhibited $50 \%$ suppression of plasma androgens after dexamethasone administration (14).

These studies suggest the existence of an ovarian androgen-producing cell with sensitivity to a pituitary hormone, similar to the postulated adrenal androgenstimulating hormone, which, indeed, displays a glucocorticoid feedback mechanism (19)'.

In the overwhelming majority of our patients, androgen concentrations were significantly decreased following the administration of dexamethasone, with no response to human chorionic gonadotropin. These findings are also quite compatible with the presence of an androgen-secreting cell in either the adrenals or ovaries, with dependence on andrenocorticotropic hormone or another pituitary androgen-stimulating hormone. Sixty percent of the patients with elevated androgens responded with varying degrees of diminished rates of hair growth. These results are comparable to those reported by Moore et al. (13). The lack of correlation between the response to treatment and the results of short-term dynamic tests has been described previously $(13,20)$.

The results in this study should be contrasted with the urinary steroid profiles reported in 24 other females with idiopathic hirsutism (21): ten to hundredfold increases in the excretion of dehydroepiandrosterone were demonstrated in over $50 \%$ of these patients. Dehydroepiandrosterone levels were suppressed much more than other steroids following administration of dexamethasone. In our series of patients with idiopathic hirsutism elevations of androsterone and aetiocholanolone, rather than dehydroepiandrosterone, were most frequent. Elevations of dehydroepiandrosterone in combinations with androsterone and aetiocholanolone were seen in $23 \%$. Isolated elevation of dehydroepiandrosterone was seen in only $6 \%$ of the group. Following dexamethasone administration, suppression of all steroid metabolites was noted rather than that of dehydroepiandrosterone alone.

The excretion of dehydroepiandrosterone is $<1.0 \mathrm{mg} /$ $24 \mathrm{~h}$ in normal women, with a range from 0.1 to 1.0 $\mathrm{mg} / 24 \mathrm{~h}$. Elevation of dehydroepiandrosterone has been observed in stress in normal subjects (22). This raises the question of whether the elevations of dehydroepiandrosterone noted in our hirsute females were manifestations of stress. This seems unlikely, since the elevations of dehydroepiandrosterone were accompanied by elevations of androsterone or aetiocholanolone in all but 2 of our subjects. It therefore appears that all these steroid metabolites showing elevated levels are associated with the presence of 
hirsutism. When dehydroepiandrosterone is the only urinary 17-ketosteroid excreted in elevated quantities, stress may be an alternative explanation, rather than reduced adrenal $3 \beta$-hydroxysteroid dehydrogenase activity. The possibility of an enzymatic block can be verified by stimulation with adrenocorticotropic hormone (8).

In summary, fractionation of the 17 -ketosteroids by gas chromatography seems to be an efficient method for detecting the presence of hyperandrogenicity and in assessing its origin. When used in conjunction with

\section{References}

1. Toaff, R., Toaff, M. E., Gould, S. \& Chayen, R. (1979) Fertil. Steril. 29, 407-413.

2. Lorenzo, E. M. (1970) J. Clin. Endocrinol. Metab. 31, $556-564$.

3. Cope, C. L. (1972) Adrenal steroids and disease (pp. 105119) JB Lippincott Company, Philadelphia.

4. Gould, S., Chayen, R., Harell, A., Toaff, M. E. \& Toaff, R. (1975) Isr. J. Med. Sci. 11, 1219-1220.

5. Vermeulen, A. \& Rubens, R. (1979) In: The Adrenal Gland (James, H. T., ed.) pp. 254-282, Raven Press, New York.

6. Abraham, G. E. (1974) J. Clin. Endocrinol. Metab. 39, $340-346$.

7. Bongiovanni, A. N. (1961) Clin. Endocrinol. 21, 860-862.

8. Bongiovanni, A. N. (1983) J. Steroid Biochem. 18, 745747.

9. Gower, D. B. (1984) In: Biochemistry of Steroid Hormones (Makin, H. L. J., ed.) pp. 349-382. Blackwell Scientific Publications, Oxford.

10. Baird, D. T. (1974) Eur. J. Obstet. Gynecol. Reprod. Biol. 4, 31-39.

11. Serafini, P., Alban, F. \& Lobo, R. A. (1985) J. Clin. Endocrinol. Metab. 60, 349-355. dynamic studies, it can detect the presence of adrenocorticotropic hormone or human chorionic gonadotropin dependency. Elevated concentrations of dehydroepiandrosterone may indicate the presence of a $3 \beta$-hydroxysteroid dehydrogenase deficiency as the basis for hyperandrogenicity.

\section{Acknowledgements}

We are grateful to $E$. Weiss for performing the radioimmunoassays. We thank Chaya Goldfarb and Shoshana Ludvinovsky for performing the gas chromatography determinations.

12. Guthrie, G. P., Wilson, E. A., Quillen, D. L. \& Jawad, M. J. (1982) Arch. Intern. Med. 142, 729-735.

13. Moore, A., Magee, F., Cunningham, S., Culliton, M. \& McKenna, T. J. (1983) Clin. Endocrinol. 18, 391-399.

14. Kirschner, M. A. \& Jacobs, J. B. (1971) J. Clin. Endocrinol. Metab. 33, 199-209.

15. Andre, C. M. \& James, V. H. T. (1974) Steroids 24, 295300.

16. Kirschner, M. A., Zucker, I. R. \& Jespersen, D. (1976) N. Engl. J. Med. 294, 637-640.

17. Lissek, K., Schurenkamper, P., Freidrich, W. \& Rutkowsky, J. (1980) Acta Endocrinol. 93, 216-222.

18. Reforzo-Membrives, J., Rocco, D. Z., Enriora, C. L. \& Mazza, S. C. (1967) Acta. Endocrinol. 55, 685-696.

19. Parker, L. N. \& Odell, W. D. (1980) Endocrine Reviews $I$, 392-410.

20. Ettinger, B., Goldfield, E. E., Burwill, K. C., Von Werder, K. \& Forsham, P. (1975) Am. J. Med. 54, 195-200.

21. Egger, H. J., Reiner, J. \& Spiteller, G. (1978) J. Chromatogr. 145, 359-369.

22. Pfeifer, P. \& Spiteller, G. (1981) J. Chromatogr. 223, $21-32$.

Moshe M. Werber

BioTechnology General

Kiryat Weizmann

76326 Rehovot

Israel 


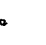

